\title{
ACTIVE NOISE CONTROL DESIGN WITH SIMPLE GENETIC ALGORITHM
}

\author{
N. Jafferi and M. O. Tokhi \\ Department of Automatic Control and Systems Engineering, \\ The University of Sheffield, UK \\ E-mail: cop03nj@sheffield.ac.uk
}

\section{KEYWORDS:}

Active noise control, genetic algorithm, noise cancellation.

\begin{abstract}
This paper presents an investigation into the development of a fixed controller for noise cancellation using a simple genetic algorithm (GA). A single-input single-output (SISO) configuration of the control algorithm is developed on the basis of optimum cancellation of noise at an observation point. The algorithm is implemented on a GA and its performance assessed in the cancellation of noise in a free-field medium. The global search technique of GA is used to develop an active noise control (ANC) system and evaluate its performance. In this investigation, randomly selected parameters are optimised for different, arbitrarily chosen coordinates to fit the system by applying the working mechanism of GA. The percentage of reinforcement that occurs in the medium is adopted as the objective function value. GA is utilised to obtain the best controller parameters among all the attempted orders and configurations. The GA-ANC algorithm is implemented and simulation results are presented to asses the performance of the system, with singlefrequency noise.
\end{abstract}

\section{INTRODUCTION}

Active noise control consists of generating cancellation source(s) to destructively interfere with the noise and thus cancel the noise level at observation point(s). This is realized by detecting the noise and processing this via a suitable electronic controller (Nelson and Elliot 2000; Tokhi and Leitch 1992; Tokhi and Veres 2002). This paper presents an investigation into the development of an ANC mechanism within a fixed controller framework. An ANC system is designed utilizing a SISO control structure to yield optimum cancellation at an observation point in a free-field propagation medium. The performance results are presented in a graphical user interface to allow interaction between the environment and the user and assessment of the system performance.
In this work, the concept of parametric analysis of the field cancellation process (Tokhi and Leitch 1992; Tokhi and Veres 2002) is used. This is a frequency-domain analysis approach based on power spectral density functions. Derivation of the algorithm has previously been reported (Tokhi and Leitch 1992; Tokhi and Veres 2002).

Genetic algorithm is a population based global search procedure inspired by the laws of natural selection and genetics (Holland 1975). The GA evolves a collection of elements called population of individuals. Each individual of the population represents a trial solution to the problem of optimizing a given fitness function. The standard simple GA works to randomly generate an initial population. Genetic inheritance operations based on selection, mating and mutation are performed to generate "offspring" that compete for survival to make up the next generation (Mitchell 1998).

\section{ACTIVE NOISE CONTROL}

A schematic diagram of the geometric arrangement of a SISO ANC structure is shown in Figure 1(a). The unwanted primary signal is detected, then processed by a controller of suitable frequency-dependent characteristics and fed to a secondary source. The secondary signals thus generated interfere with the primary signal to cancel the noise at an observation point in the medium.

A frequency-domain equivalent block diagram of the ANC structure is shown in Figure 1(b) where, E(s), F(s), $\mathrm{G}(\mathrm{s})$ and $\mathrm{H}(\mathrm{s})$ represent transfer characteristics of the propagation paths between the primary source and the detector, secondary source and the detector, primary source and the observer and secondary source and the observer respectively. $\mathrm{M}(\mathrm{s}), \mathrm{C}(\mathrm{s})$ and $\mathrm{L}(\mathrm{s})$ represent transfer characteristics of the detectors, controller and secondary sources respectively. $\mathrm{D}(\mathrm{s}), \mathrm{P}(\mathrm{s}), \mathrm{Po}(\mathrm{s}), \mathrm{S}(\mathrm{s})$, $\mathrm{So}(\mathrm{s})$ and $\mathrm{O}(\mathrm{s})$ represent the detected signals, primary signal at the source and observation points, secondary signals at the source and observation points and the combined primary and secondary (observed) signal at the observation point respectively.

The objective in Figure 1 is to achieve full noise cancellation at the observation point. This requires the 
primary and secondary signals at the observation point to be equal in amplitudes and have a phase difference of $180^{\circ}$ relative to one another. This in terms of the block diagram in Figure 1(b) gives

$$
\mathrm{O}(\mathrm{s})=\mathrm{So}(\mathrm{s})+\mathrm{Po}(\mathrm{s})=0
$$

Using the block diagram in Figure 1(b) to obtain Po(s) and $\mathrm{So}(\mathrm{s})$, substituting into equation (1) and solving for $\mathrm{C}(\mathrm{s})$ yields

$$
\mathrm{C}(\mathrm{s})=\mathrm{M}(\mathrm{s})^{-1} \Delta(\mathrm{s})^{-1} \mathrm{G}(\mathrm{s}) \mathrm{H}(\mathrm{s})^{-1} \mathrm{~L}(\mathrm{~s})^{-1}
$$

where $\Delta(\mathrm{s})$ is a matrix given by

$$
\Delta(\mathrm{s})=\left[\mathrm{G}(\mathrm{s}) \mathrm{H}(\mathrm{s})^{-1} \mathrm{~F}(\mathrm{~s})-\mathrm{E}(\mathrm{s})\right]
$$

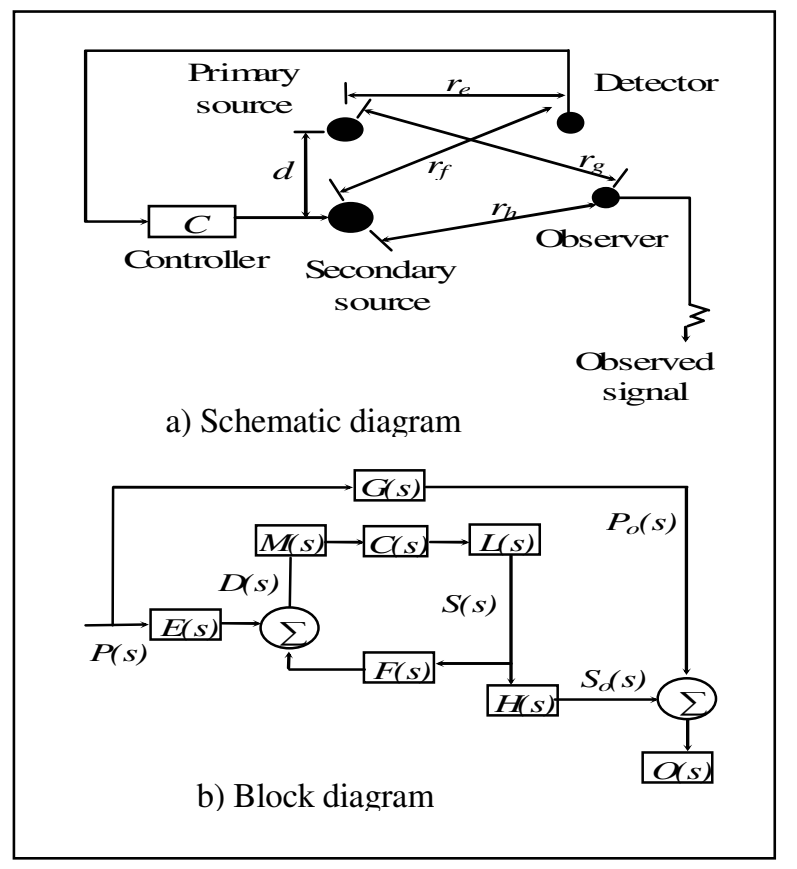

Figure 1: Feedforward control structure

Equation (2) is the required controller transfer function for optimum cancellation of noise at the observation point. The characteristics of the controller are determined by the geometric arrangement of system components.

The interference of the component waves through the process of implementing of the ANC system will lead to a pattern of zones of cancellation and reinforcement in the medium; a region of cancellation is created around the observation point, and reinforcement will occur in regions further away from this point. The physical extent of zones of cancellation depends on the maximum frequency of the noise and separation between the sources.

A continuous frequency-dependent controller characteristic given in equation (2) within the ANC system of Figure 1(b) will ensure cancellation of the noise at and in the surrounding of the observation point.

Using Figure 1(b), the secondary signal S(s) can be expressed as

$$
\mathrm{S}(\mathrm{s})=[\mathrm{P}(\mathrm{s}) \mathrm{E}(\mathrm{s})+\mathrm{F}(\mathrm{s}) \mathrm{S}(\mathrm{s})] \mathrm{M}(\mathrm{s}) \mathrm{C}(\mathrm{s}) \mathrm{L}(\mathrm{s})
$$

Substituting for $\mathrm{C}(\mathrm{s})$ from equation (2) into equation (4), using equation ( 3 ) and simplifying, $s=j \omega$, yields:

$$
S(j \omega)=-P(j \omega) G(j \omega) H(j \omega)^{-1}
$$

Consider an arbitrary point $\mathrm{q}$ in the medium and let

$P(j \omega)$ represent the wave emitted by the primary source in the frequency domain,

$\mathrm{S}(\mathrm{j} \omega)$ represent the wave emitted by the secondary source in the frequency domain,

$\operatorname{Po}(j \omega)$ represent $\operatorname{Po}(s)$ in the frequency domain,

$\operatorname{So}(j \omega)$ represent $\mathrm{So}(\mathrm{s})$ in the frequency domain,

$\mathrm{g}_{\mathrm{q}}(\mathrm{j} \omega)$ represent transfer characteristics of acoustic path between the primary source and the arbitrary point q,

$h_{q}(j \omega)$ represent transfer characteristics of acoustic path between the secondary source and the arbitrary point $q$,

$\mathrm{S}_{\mathrm{yyD}}(\omega)$ represent the autopower spectral density of primary wave at the arbitrary point $\mathrm{q}$,

$S_{y y q}(\omega)$ represent the autopower spectral density of secondary wave at the arbitrary point q.

The transfer characteristics $g_{q}(j \omega)$ and $h_{q}(j \omega)$ for the propagation medium under consideration are given by:

$$
\begin{aligned}
& g_{q}(j \omega)=\frac{A}{r_{g q}} e^{-j \frac{\omega}{c} r_{g q}} \\
& h_{q}(j \omega)=\frac{A}{r_{h q}} e^{-j \frac{\omega}{c} r_{h q}}
\end{aligned}
$$

where A is a constant, $\mathrm{c}$ is the speed of sound in the medium, $r_{\mathrm{gq}}$ is the distance between the primary source and arbitrary point $\mathrm{q}$ and $\mathrm{r}_{\mathrm{hq}}$ is the distance between the secondary source and arbitrary point q.

The autopower spectral densities of the primary and secondary waves at the arbitrary point can be written as:

$$
\begin{aligned}
& S_{y y D}(\omega)=|P o(j \omega)|^{2}=\left|P(j \omega) g_{q}(j \omega)\right|^{2} \\
& S_{y y q}(\omega)=|S o(j \omega)|^{2}=\left|P(j \omega) g_{q}(j \omega)+S(j \omega) h_{q}(j \omega)\right|^{2}
\end{aligned}
$$

For cancellation of the primary wave to occur at the arbitrary point $\mathrm{q}, \mathrm{S}_{\mathrm{yyq}}(\omega)$ should be less than $\mathrm{S}_{\mathrm{yyD}}(\omega)$;

$$
\mathrm{S}_{\mathrm{yyq}}(\omega)<\mathrm{S}_{\mathrm{yyD}}(\omega)
$$

For a quantitative description of cancellation, the field cancellation factor $\mathrm{K}$ is defined as the ratio of the cancelled spectrum $S_{\text {yyq }}(\omega)-S_{y y D}(\omega)$ to the primary spectrum $S_{y y D}(\omega)$ that existed at the point prior to 
introducing the secondary wave (Tokhi and Leitch 1992):

$$
K \stackrel{\Delta}{=} 1-\frac{S_{y y q}(\omega)}{S_{y y D}(\omega)}
$$

It follows form equations (8) and (9) that, for the primary wave to be cancelled, $\mathrm{K}$ must be between zero and unity:

$$
0<\mathrm{K} \leq 1
$$

where $\mathrm{K}=1$ means complete cancellation, $\mathrm{K}=0$ means no cancellation and $\mathrm{K}<0$ means that reinforcement will occur.

Substituting for $S(j \omega)$ from equation (5) into equation (7), simplifying and using equation (9) yields the cancellation factor $\mathrm{K}$ at the arbitrary point $\mathrm{q}$ in the medium as:

$$
\mathrm{K}=1-\left|1+\mathrm{g}_{\mathrm{q}}{ }^{-1}(\mathrm{j} \omega) \mathrm{G}(\mathrm{j} \omega) \mathrm{H}^{-1}(\mathrm{j} \omega) \mathrm{h}_{\mathrm{q}}(\mathrm{j} \omega)\right|^{2}
$$

Equation (11) gives a quantitative measure of the degree of cancellation achieved with the ANC system at the arbitrary point $\mathrm{q}$ under stationary conditions.

\section{GENETIC ALGORITHMS}

In this investigation, randomly selected parameters are optimised for different, arbitrarily chosen coordinates of system components by applying the working mechanisms of GA as explained above. The aim is to minimise the percentage of the whole medium that corresponds to reinforcement in the ANC system, defined as a problem to which this method can be applied. This is done using equation (11) to calculate the cancellation factor $\mathrm{K}$ in each segment of the medium and referring the result to equation (10).

If the cancellation factor, $\mathrm{K}$ happens to be more than or equal to 0 , this means that cancellation occurs in that segment, but if the cancellation factor $\mathrm{K}$ is less than 0 , the region of reinforcement is indeed created there. Every reinforcement region is counted and for a quantitative ratio description of cancellation, the sum of field cancellation factor $\mathrm{K}$ is defined as:

$$
\% \text { Cancellation }=\frac{100-\text { Sum of } \mathrm{XX}}{\mathrm{YY} \times \mathrm{ZZ}} \times 100 \%
$$

where XX represents reinforcement area, YY the number of segment of length $\mathrm{X}$ in the medium and $\mathrm{ZZ}$ represents the number of segments of length $\mathrm{Y}$ in the medium.

The parameters used for the SISO ANC GA are as follows :

Number of variables: 8

Population size: 30
Maximum number of generation: 100

Mutation rate: 0.001

Crossover rate: 0.7

Target to achieve: $10 \%$

(Percentage of reinforcement to achieve)

Chromosome precision string representation: 16 bits

Crossover type: single-point

Selection type: stochastic universal sampling

Termination of the GA is applied after a specified number of generations or if the target is achieved. The results are tested against the problem definition. If no acceptable solutions are found, the GA may be restarted or a fresh search initiated.

\section{SIMULATION RESULTS}

A simulation environment was developed using MATLAB, C programming language (Hanselman and Littlefield 2001) and Genetic Algorithm TOOLBOX (Chipperfield et al. 1994). The simulation incorporates development of a fixed controller for noise cancellation using GA. A SISO control configuration is used for optimum cancellation of noise at an observation point.

A SISO ANC system was set up on a 2D coordinate system with primary source at $(-1.046 \mathrm{~m},-1.915 \mathrm{~m})$, secondary source at $(-1.270 \mathrm{~m},-1.557 \mathrm{~m})$, detector at $(1.158 \mathrm{~m}, 0.472 \mathrm{~m})$ and observer at $(-0.378 \mathrm{~m}, 1.381 \mathrm{~m})$, and the GA was run for a primary wave of $340 \mathrm{~Hz}$. Figure 2 shows the performance of the system in 3D and 2D after the first generation, where cancellation is indicated in the 2D contour graph with blue colour and relative sound level between 0 and 1 in 3D graph. The result shows that about $55 \%$ cancellation was achieved over the whole area.

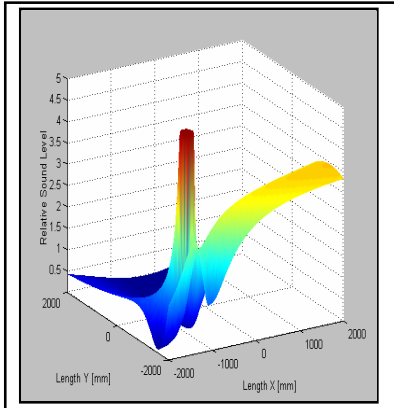

a) $3 \mathrm{D}$ graph

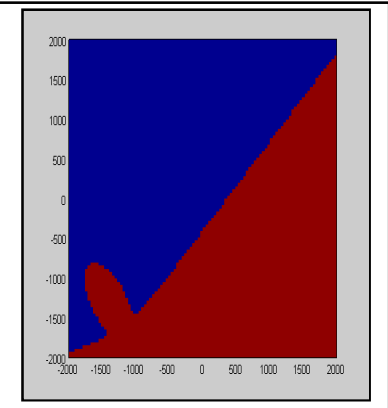

b) Contour graph
Figure 2: Interference pattern created by the SISO GAANC after the first generation.

Figure 3 shows the convergence graph of the GA search process with a target of achieving no more than $10 \%$ reinforcement in the region. 


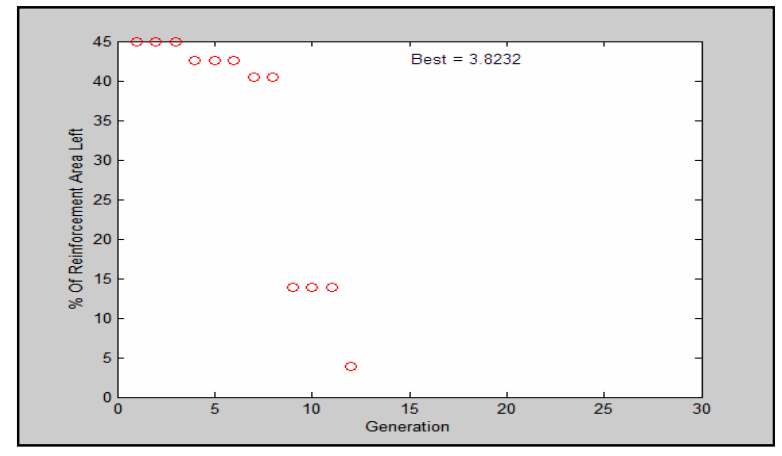

Figure 3: Convergence graph of SISO GA-ANC

Figure 4 shows the performance of the SISO GA-ANC with system set up with the primary source at $(-1.046 \mathrm{~m}$, $-1.915 \mathrm{~m})$, secondary source at $(-1.270 \mathrm{~m},-1.557 \mathrm{~m})$, detector at $(1.158 \mathrm{~m}, 0.472 \mathrm{~m})$ and observer at $(-0.378 \mathrm{~m}$, $1.381 \mathrm{~m}$ ) and noise frequency of $340 \mathrm{~Hz}$. The result shows that the best configuration results from randomising the selection of locations of system components; coordination found the best optimisation at a generation 22 with nearly $96 \%$ cancellation in whole area.

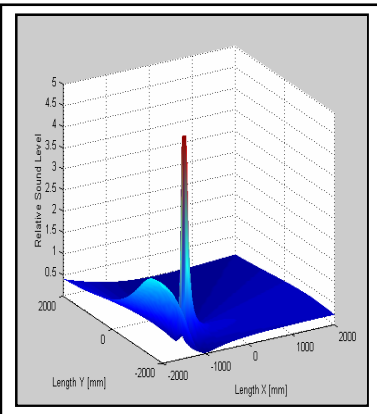

a) $3 \mathrm{D}$ graph

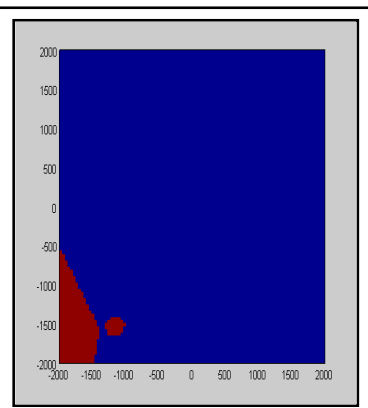

b) Contour graph
Figure 4: Performance of SISO GA-ANC at first generation with a $340 \mathrm{~Hz}$ noise frequency.

The simulation environment has been developed using MATLAB and C programming language. It divided into several sections with the following features:

1) Selection of noise frequency.

2) Geometrical configuration of system components for the first and final generations of the GA.

3) GA parameter configuration.

4) Display of system performance with choices of 3D, contour and 2D graph.

5) The resulting percentage of cancellation area and the corresponding generation number.

6) Convergence graph of the GA

The graphical user interface of the developed environment is shown in Figure 5, where the regions indicated by circled numbers correspond to the features listed above.

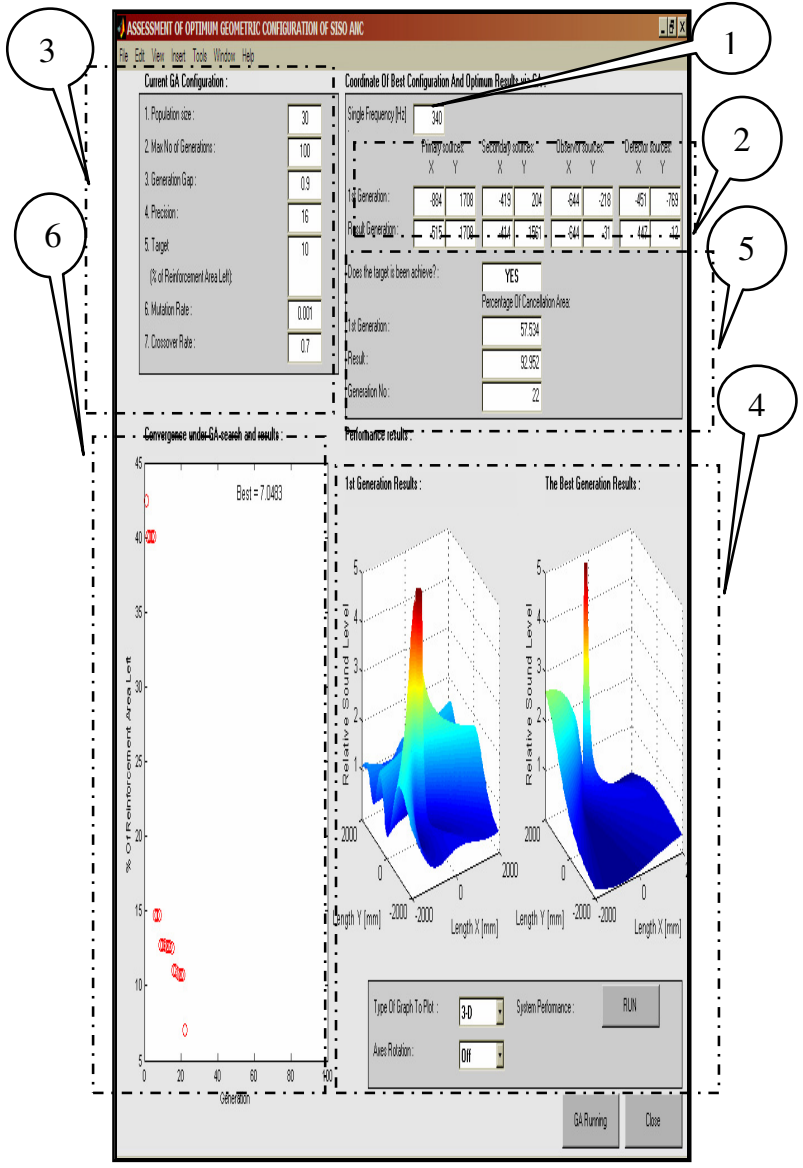

Figure 5: Overall interface of the system

Figure 6 shows an overall interface of assessment of geometrical configuration of SISO GA-ANC using with a $340 \mathrm{~Hz}$ frequency, population size of 30 , maximum generation set to 100 and target to achieve set as no more than $10 \%$ reinforcement. The result shows that the best configuration results from randomising the locations of system components. The best optimised configuration was reached at generation 15 with region of cancellation constituting nearly $92.7 \%$ of the whole area.

Table 1 shows the average cancellation in percentage of the whole region at the first generation and at generation number where the GA process was stopped. Each exercise was run 100 times and the average over the 100 trials has been displayed in each case.

Table 1: Average cancellation achieved for three cases.

\begin{tabular}{|l|l|l|l|}
\hline $\begin{array}{l}\text { Target } \\
\text { reinforcement area }\end{array}$ & $0.1 \%$ & $1 \%$ & $10 \%$ \\
\hline $\begin{array}{l}\text { Cancellation } \\
\text { region after the } \\
\text { first generation }\end{array}$ & $69.90 \%$ & $65.55 \%$ & $65.95 \%$ \\
\hline $\begin{array}{l}\text { Average } \\
\text { cancellation after } \\
\text { last generation }\end{array}$ & 47.16 & 27.42 & 15.54 \\
\hline
\end{tabular}



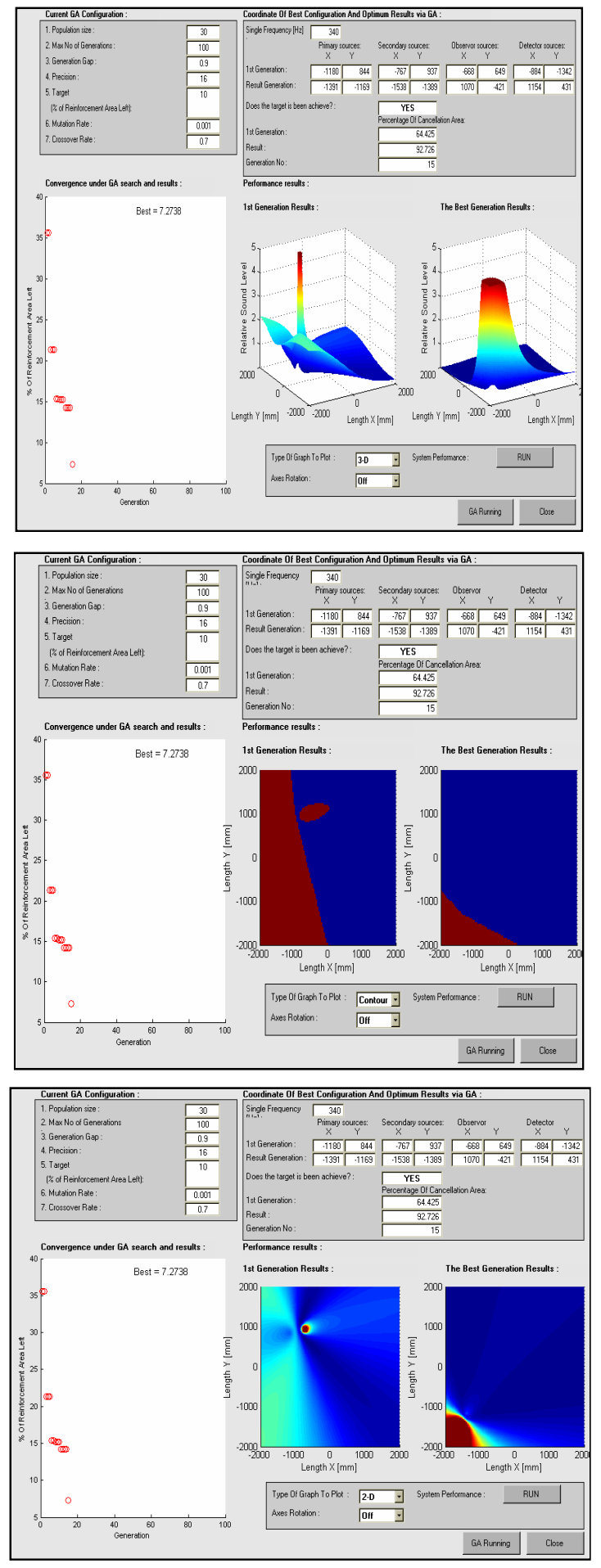

Figure 6: System interface for assessment of optimum geometric configuration of SISO ANC

It was noted that the bigger the target value, the smaller the generation number achieving the target. For a target of $10 \%$ the GA achieved that target at about 15 to 20 generations.

Table 2 shows the average cancellation region in the first generation and last generation for two cases of maximum number of generations. In each case the GA was run 100 times and the average of the results obtained.

Table 2: Average cancellation for specified maximum number of generations

\begin{tabular}{|l|c|c|}
\hline $\begin{array}{l}\text { Maximum number of } \\
\text { generations }\end{array}$ & 20 & 100 \\
\hline $\begin{array}{l}\text { Cancellation region } \\
\text { after first generation }\end{array}$ & $68.68 \%$ & $64.00 \%$ \\
\hline $\begin{array}{l}\text { Cancellation region } \\
\text { after last generation }\end{array}$ & $92.48 \%$ & $94.90 \%$ \\
\hline
\end{tabular}

It is noted that the longer the GA is run, the better the result will be. However, note that GA takes long time, and it is recommended to run the GA only to the extent that acceptable results are obtained.

\section{CONCLUSIONS}

The design and implementation of an ANC system using GA has been presented and verified through simulated exercises. It has been shown that by using the minimum information specific to the system which may be easily estimated using the system's input and output together with certain trial and error experiments, optimisation for good control performance gains can be achieved with GAs. Future work will look into extending the approach to multiple source configurations.

\section{REFERENCES}

Chipperfield, A. J.; P.J. Fleming; H. Pohlheim; and C. Fonseca. 1994. "A genetic algorithm toolbox for MATLAB". In Proceedings of the International Conference on Systems Engineering (Coventry, UK, 06-08 September).

Hanselman, D. and B. Littlefield. 2001. Mastering MATLAB 6, A comprehensive Tutorial and Reference, Prentice Hall.

Holland, J. 1975. Adaptation in natural and artificial systems, University of Michigan Press, USA.

Mitchell, M. 1998. An Introduction to Genetics Algorithms, The MIT Press, London, England.

Nelson, P. A. and S. J. Elliot. 2000. Active Control of Sound, Academic Press, London.

Tokhi, M. O. and R. R. Leitch. 1992. Active Noise Control, Clarendon Press, Oxford.

Tokhi, M. O. and S. Veres. 2002. Active Sound and Vibration Control, theory and applications, The Institute of Electrical Engineers, Michael Faraday House, Herts. 


\section{AUTHOR BIOGRAPHIES}

Noormaziah Jafferi was born in Johor, Malaysia and went to Takushoku University, where she studied mechanical systems and obtained her degree in 2002. She is now a research student at The University of Sheffield.

Dr. M. O. Tokhi is a Reader at the University of Sheffield. His research interests include active control of noise and vibration, adaptive/intelligent and soft computing approaches for modelling and control of dynamic systems, high-performance real-time computing and biomedical applications of robotics and control. Further details may be found at the url: http://www.shef.ac.uk/acse/staff/mot 